\title{
Dry Wear Behavior of Heat Treated Eutectic Al-Si Alloy
}

\author{
Sridhara T \\ ${ }^{1}$ Asst. Professor and Research Scholar, Department of Mechanical Engineering, SDM Institute of \\ Technology, Ujire-574240, Karnataka, India \\ Dr. H. R. Manohara \\ ${ }^{2}$ Professor and Head R\&D, Department of Mechanical Engineering, SJM Institute of Technology, \\ Chitradurga-577502, Karnataka, India \\ Dr. Basava T \\ ${ }^{3}$ Professor and Head IEDC, Department of Mechanical Engineering, SDM Institute of Technology-574240, \\ Ujire, Karnataka, India
}

\begin{abstract}
A Eutectic Al-Si alloy (A413) was reinforced with varied percentage of intermetallic particles like $\mathrm{Al}_{3} \mathrm{Ti}$, $\mathrm{AlB}_{2}$, $\mathrm{TiB}_{2}$ and $\mathrm{Al}_{4} \mathrm{Sr}$ particles by stir casting using graphite moulds. They were heat treated in induction furnace. The Pin-onDisc apparatus was used to study Wear behavior at different sliding speeds, pressures, sliding distance and heat treatment. Heat treatment resulted in further improvement in wear resistance. It is concluded that the intermetallic particles influences on wear rate. The higher temperature reveals the wear resistance due to the glaze formation that offers a protection and avoids further wear. Due to combined reinforced alloys to A413 alloy has resulted in minimum weight loss when compared to the individual addition $\mathrm{Al}_{3} \mathrm{Ti}, \quad \mathrm{AlB}_{2}, \mathbf{T i B}_{2}$ and $\mathbf{A l}_{4} \mathrm{Sr}$ reinforced intermetallic alloys and in heat treated conditions.
\end{abstract}

Key words: Composites, Eutectic Alloy, Wear behavior, Heat treatment.

\section{INTRODUCTION}

Since the early dawn of civilization, the strong and light material has always fascinated mankind for typical applications. An aluminium casting has low strength and hardness and poor machinability [1-6]. With alloying elements like silicon, magnesium and to a lesser extent copper, titanium, boron and strontium improve the properties [4-5]. Silicon is an important addition to aluminium because of its excellent characteristics and these are high strength to weight ratio, excellent castability, pressure tightness, and low co-efficient of thermal expansion, good thermal conductivity, good mechanical properties and corrosion resistance [6-12]. Minor additions of titanium, boron and are normally used for grain refinement and strontium is used for the modification of eutectic silicon [1218]. Eutectic alloys (A413) [10-13\% silicon] are used for pistons, cylinders, blocks and heads of IC engines in automobile and aeronautical industries [18-27].

There is limited study concerning the heat treated sliding wear properties of Al-Si alloys reinforced with $\mathrm{Al}-\mathrm{Al} 3 \mathrm{Ti}$, $\mathrm{Al}-\mathrm{AlB} 2, \mathrm{Al}-\mathrm{TiB} 2$ and Al-Al4Sr intermetallic particles. The latter are mostly used for automobile components like piston and cylinder blocks which operate in general at higher temperatures. It was understood that it is difficult to make a quantitative comparison of the literature data since the wear rate and friction coefficient strongly depends on the test methods, test environment, testing variables, composite manufacturing route and the reinforcement volume fraction, size and hardness [28-31].

In this work to achieve additional improvements in wear resistance, the intermetallic particles of $\mathrm{Al}_{3} \mathrm{Ti}_{1} \mathrm{TiB}_{2}, \mathrm{AlB}_{2}$ and $\mathrm{Al}_{4} \mathrm{Sr}$ were added to A413alloy, and sliding wear tests were carried out under dry sliding conditions at room temperature with heat treated.

\section{EXPERIMENTAL}

The alloy selected for the study is Eutectic Al-Si alloy (A413). The optimized compositions of alloys specimens are prepared for the test is as shown in Table 1. These composite have been studied for their dry wear behavior at room temperature. Melting was carried out in an induction furnace with a graphite crucible held at a temperature of 720 ${ }^{0} \mathrm{C}$. After degassing with solid hexachlorothane $\left(\mathrm{C}_{2} \mathrm{Cl}_{6}\right)$, reinforced optimized intermetallic particle alloy chips (Al$5 \% \mathrm{Al}_{3} \mathrm{Ti}, \mathrm{Al}-3 \% \mathrm{AlB}_{2}, \mathrm{Al}-3 \% \mathrm{TiB}_{2}$ and $\mathrm{Al}-10 \% \mathrm{Al}_{4} \mathrm{Sr}$ ) were added to the melt for grain reinforcement. The melt was 
stirred for 30s after adding the grain refiner, held for $5 \mathrm{~min}$ and poured into preheated graphite mould to obtain the casting diameter of $12.5 \mathrm{~mm}$ and height $125 \mathrm{~mm}$. The cast bars were further heat treated in muffle furnace for 120 minutes at $50{ }^{\circ} \mathrm{C}$ and natural cooling was done. The cast bars were turned into $10 \mathrm{~mm}$ diameter and $40 \mathrm{~mm}$ length for wear test.

Table 1. The optimized compositions of A413 alloy

\begin{tabular}{|c|c|l|c|}
\hline Sl. No. & Alloy no. & \multicolumn{1}{|c|}{ Alloy Composition } & Melt holding time (min) \\
\hline 1 & $\mathrm{~A}$ & $\mathrm{~A} 413$ & $0 *$ \\
\hline 2 & $\mathrm{~B}$ & $\mathrm{~A} 413+0.27 \%\left(\mathrm{Al}-5 \% \mathrm{Al}_{3} \mathrm{Ti}\right)$ & 5 \\
\hline 3 & $\mathrm{C}$ & $\mathrm{A} 413+0.6 \%\left(\mathrm{Al}-3 \% \mathrm{AlB}_{2}\right)$ & 5 \\
\hline 4 & $\mathrm{D}$ & $\mathrm{A} 413+0.55 \%\left(\mathrm{Al}-3 \% \mathrm{TiB}_{2}\right)$ & 5 \\
\hline 5 & $\mathrm{E}$ & $\mathrm{A} 413+0.3 \%\left(\mathrm{Al}-10 \% \mathrm{Al}_{4} \mathrm{Sr}\right)$ & 5 \\
\hline 6 & $\mathrm{~F}$ & $\begin{array}{l}\mathrm{A} 413+0.55 \%\left(\mathrm{Al}-3 \% \mathrm{TiB}_{2}\right) \\
+0.3 \%(\mathrm{Al}-10 \% \mathrm{Al} 4 \mathrm{Sr})\end{array}$ & $* 0$ " means No holding time \\
\hline
\end{tabular}

The experiments were conducted according to a factorial design, W. E. Duckworth, Statistical Techniques in Technological Research; Aid to Research Productivity, Methuen, London, 1968. The experimental parameters are referred to as factors and different chosen values of each parameter are referred to as different levels of that factor. The experiments are carried out in such a way that no two experiments are identical in one set. However, the whole set is replicated to evaluate the errors involved in the measurements. The factors chosen for study are 6 samples i.e. intermetallic content, bearing pressure (3 levels), speed ( 3 levels) \& sliding distance ( 3 levels) as shown in Table 2. The following are the different levels for each of the experimental parameter.

Table2. Experimental parameters

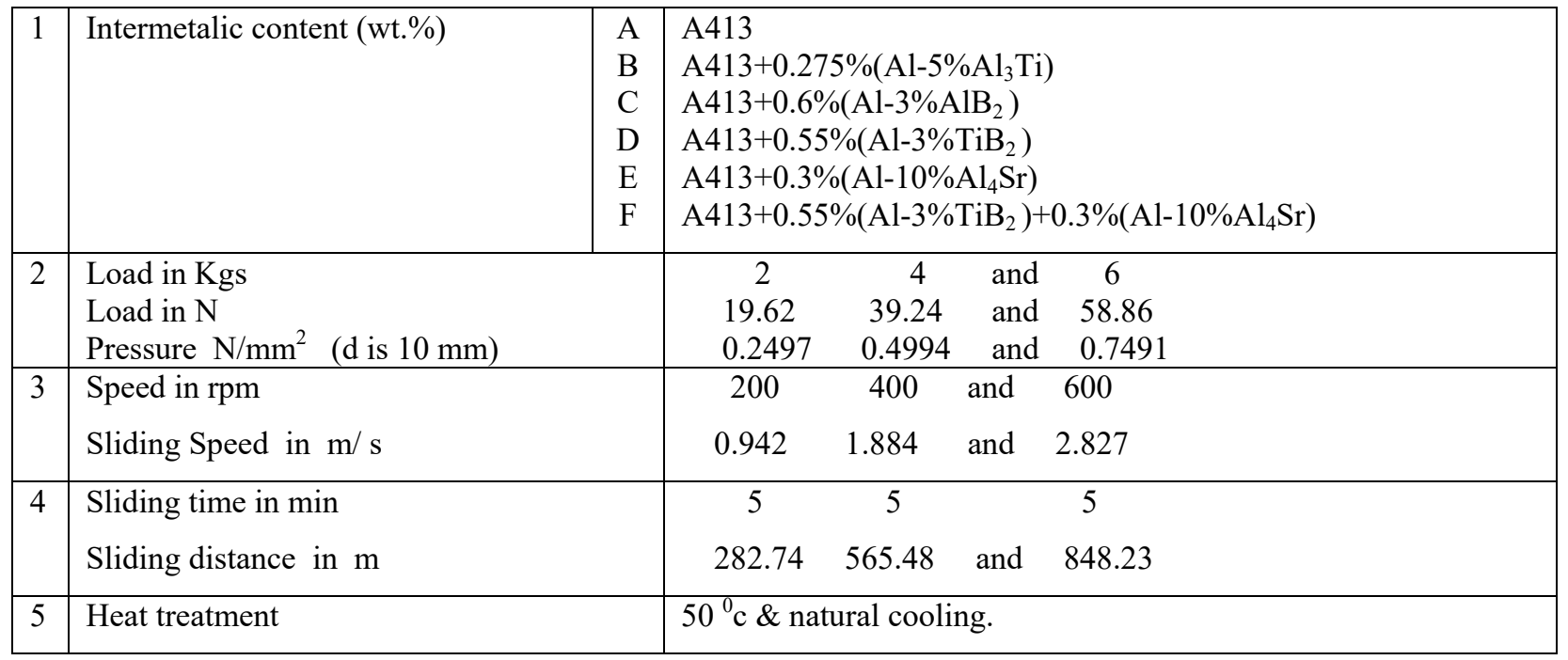

\section{RESULTS \& DISCUSSIONS}

\section{a. $\quad$ SLIDING WEAR BEHAVIOR OF AL-SI ALLOY (A413 ALLOY)}

Wear, the progressive damage and material loss, occurs on the surface of a component as a result of its motion relative to the adjacent working parts. In the present work the effect of Al-Ti-B grain refiner and Al-Sr modifier on the wear behavior of eutectic Al-Si alloy have been studied with various process parameter, in addition an attempt has been made to throw some light on the mechanism of Al-Si alloys before and after the wear studies. Sliding wear tests were carried out using Pin-on-Disc (Ducom TR-20-PHM-400) type wear testing machine, with a large number of variables. Which affect the wear mechanism and wear rate. 


\section{b. EFFECT OF LOAD}

The effect of grain reinforcement on weight loss of A413 alloy under different load with constant sliding speed and at constant sliding distance. The effect of load on volumetric wear of A413 alloy under constant sliding speed $(0.942,1.884$ and $2.827 \mathrm{~m} / \mathrm{sec})$ and at constant sliding distance $(282.74,565.48$, and $848.23 \mathrm{~m})$. From figures it is clear that the weight loss of A413 alloy increases with increase in load in all the cases studied and the wear was higher in case of untreated A413 alloy, i.e. addition of intermetallic alloys show less volumetric wear as compared to the absence of grain refiner. It is clear from the Table 3 \& figure 1, A413 (A1) of load v/s wear rate for $200 \mathrm{rpm}$ shows that the wear rate of $\left(6.5431 \times 10^{-6} \mathrm{~g} / \mathrm{m}\right)$ at load $20 \mathrm{~N}$, as the load increases then the wear rate also increases to $\left(25.4650 \times 10^{-6} \mathrm{~g} / \mathrm{m}\right)$ at load $60 \mathrm{~N}$ as they do not have intermetallic particles and wear tends to increase. $\mathrm{A} 413+5 \% \mathrm{Al}_{3} \mathrm{Ti}(\mathrm{B} 1), \mathrm{A} 413+3 \% \mathrm{AlB}_{2}(\mathrm{C1}), \mathrm{A} 413+10 \% \mathrm{Al}$ Sr (E1) of load v/s wear rate for $200 \mathrm{rpm}$ which shows that the wear rate decreases slightly as load increases by adding the intermetallic particles. $\mathrm{A} 413+3 \% \mathrm{TiB}_{2}+10 \% \mathrm{Al}_{4} \mathrm{Sr}(\mathrm{F} 1)$ of load $\mathrm{v} / \mathrm{s}$ wear rate for $200 \mathrm{rpm}$ which as minimum wear rate of $\left(1.7684 \times 10^{-6} \mathrm{~g} / \mathrm{m}\right)$ at load $40 \mathrm{~N}$ shows the remarkable trend with increasing load. From Table $2 \&$ figure $2, \mathrm{~A}_{413}+3 \% \mathrm{TiB}{ }_{2}(\mathrm{D} 1)$ of load $\mathrm{v} / \mathrm{s}$ wear rate for $400 \mathrm{rpm}$ shows the maximum wear rate of $\left(6.3662 \times 10^{-6} \mathrm{~g} / \mathrm{m}\right)$ at load $60 \mathrm{~N}$, by adding intermetallic particles wear tends to be more. $\mathrm{A} 413+5 \% \mathrm{Al}_{3} \mathrm{Ti}(\mathrm{B} 1), \mathrm{A} 413+3 \% \mathrm{TiB}_{2}+10 \% \mathrm{Al}_{4} \mathrm{Sr}(\mathrm{F} 1)$, of load v/s wear rate for 400 rpm which shows that wear rate decreases slightly as load increases. $\mathrm{A} 413+3 \% \mathrm{TiB}_{2}+10 \% \mathrm{Al}_{4} \mathrm{Sr}(\mathrm{F} 1)$ of

Table 3. Wear test data at Constant Sliding speed $(\mathrm{v})=0.942 \mathrm{~m} / \mathrm{s}$, Track diameter $90 \mathrm{~mm}$, Time $5 \mathrm{~min}$ and Pin diameter $10 \mathrm{~mm}$.

\begin{tabular}{|c|c|c|c|c|c|c|}
\hline \multirow[b]{2}{*}{$\begin{array}{l}\text { Sl. } \\
\text { NO }\end{array}$} & \multirow[b]{2}{*}{ Alloy composition } & \multirow[b]{2}{*}{$\begin{array}{l}\text { Load } \\
(\mathrm{N})\end{array}$} & \multicolumn{4}{|c|}{ Wt. loss in $(\mathrm{g})$ for Heat treated at $50{ }^{\circ} \mathrm{C}$} \\
\hline & & & $\begin{array}{l}\text { Alloy } \\
\text { No. }\end{array}$ & $\begin{array}{l}\text { Speed } \\
200 \mathrm{rpm}, \\
\text { Sliding } \\
\text { distance }(\mathrm{L})= \\
282.74 \mathrm{~m}\end{array}$ & $\begin{array}{l}\text { Speed } \\
400 \mathrm{rpm}, \\
\text { Sliding } \\
\text { distance }(\mathrm{L})= \\
565.48 \mathrm{~m}\end{array}$ & $\begin{array}{l}\text { Speed } \\
600 \mathrm{rpm}, \\
\text { Sliding } \\
\text { distance }(\mathrm{L})= \\
848.23 \mathrm{~m}\end{array}$ \\
\hline 1 & A413 & & A1 & 0.0018 & 0.0015 & 0.0021 \\
\hline 2 & $\mathrm{~A} 413+0.275 \%(\mathrm{Al}-5 \mathrm{Ti})$ & & B1 & 0.0026 & 0.0016 & 0.0019 \\
\hline 3 & $\mathrm{~A} 413+0.6 \%\left(\mathrm{Al}-3 \% \mathrm{AlB}_{2}\right)$ & & $\mathrm{C} 1$ & 0.0015 & 0.0017 & 0.0016 \\
\hline 4 & $\mathrm{~A} 413+0.55 \%\left(\mathrm{Al}-3 \% \mathrm{TiB}_{2}\right)$ & 19.62 & D1 & 0.0008 & 0.0016 & 0.0027 \\
\hline 5 & $\mathrm{~A} 413+0.3 \%\left(\mathrm{Al}-10 \% \mathrm{Al}_{4} \mathrm{Sr}\right)$ & & E1 & 0.0019 & 0.0015 & 0.0033 \\
\hline 6 & $\begin{array}{l}\mathrm{A} 413+0.55 \%\left(\mathrm{Al}-3 \% \mathrm{TiB}_{2}\right)+ \\
0.3 \%\left(\mathrm{Al}-10 \% \mathrm{Al}_{4} \mathrm{Sr}\right)\end{array}$ & & $\mathrm{F} 1$ & 0.0006 & 0.0013 & 0.0029 \\
\hline 1 & A413 & & A1 & 0.0035 & 0.0017 & 0.0044 \\
\hline 2 & $\mathrm{~A} 413+0.275 \%(\mathrm{Al}-5 \mathrm{Ti})$ & & B1 & 0.0012 & 0.0010 & 0.0017 \\
\hline 3 & $\mathrm{~A} 413+0.6 \%\left(\mathrm{Al}-3 \% \mathrm{AlB}_{2}\right)$ & & $\mathrm{C} 1$ & 0.0010 & 0.0028 & 0.0018 \\
\hline 4 & $\mathrm{~A} 413+0.55 \%\left(\mathrm{Al}-3 \% \mathrm{TiB}_{2}\right)$ & 39.24 & D1 & 0.0009 & 0.0023 & 0.0035 \\
\hline 5 & $\mathrm{~A} 413+0.3 \%\left(\mathrm{Al}-10 \% \mathrm{Al}_{4} \mathrm{Sr}\right)$ & & E1 & 0.0008 & 0.0020 & 0.0037 \\
\hline 6 & $\begin{array}{l}\mathrm{A} 413+0.55 \%\left(\mathrm{Al}-3 \% \mathrm{TiB}_{2}\right)+ \\
0.3 \%\left(\mathrm{Al}-10 \% \mathrm{Al}_{4} \mathrm{Sr}\right)\end{array}$ & & $\mathrm{F} 1$ & 0.0005 & 0.0009 & 0.003 \\
\hline 1 & A413 & & A1 & 0.0070 & 0.0019 & 0.0033 \\
\hline 2 & $\mathrm{~A} 413+0.275 \%(\mathrm{Al}-5 \mathrm{Ti})$ & & B1 & 0.0014 & 0.0026 & 0.0021 \\
\hline 3 & $\mathrm{~A} 413+0.6 \%\left(\mathrm{Al}-3 \% \mathrm{AlB}_{2}\right)$ & & $\mathrm{C} 1$ & 0.0010 & 0.0024 & 0.0022 \\
\hline 4 & $\mathrm{~A} 413+0.55 \%\left(\mathrm{Al}-3 \% \mathrm{TiB}_{2}\right)$ & 58.86 & D1 & 0.0020 & 0.0036 & 0.0038 \\
\hline 5 & $\mathrm{~A} 413+0.3 \%\left(\mathrm{Al}-10 \% \mathrm{Al}_{4} \mathrm{Sr}\right)$ & & E1 & 0.0016 & 0.0016 & 0.0039 \\
\hline 6 & $\begin{array}{l}\mathrm{A} 413+0.55 \%\left(\mathrm{Al}_{-}-3 \% \mathrm{TiB}_{2}\right)+ \\
0.3 \%\left(\mathrm{Al}-10 \% \mathrm{Al}_{4} \mathrm{Sr}\right)\end{array}$ & & $\mathrm{F} 1$ & 0.0009 & 0.0017 & 0.0042 \\
\hline
\end{tabular}


load v/s wear rate for $400 \mathrm{rpm}$ which has minimum wear rate of $\left(1.6799 \times 10^{-6} \mathrm{~g} / \mathrm{m}\right)$ at load $40 \mathrm{~N}$ shows the remarkable trend with increasing load. From Table $3 \&$ figure 3, A413 (A1) of load v/s wear rate for $600 \mathrm{rpm}$ shows the maximum wear rate of $\left(5.2462 \times 10^{-6} \mathrm{~g} / \mathrm{m}\right)$ at load $40 \mathrm{~N}$, they do not have intermetallic particles and wear tends to be more. $\mathrm{A} 413+5 \% \mathrm{Al}_{3} \mathrm{Ti}(\mathrm{B} 1)$ of load $\mathrm{v} / \mathrm{s}$ wear rate for $600 \mathrm{rpm}$ shows that wear rate decreases slightly as load increases. $\mathrm{A}_{413}+3 \% \mathrm{AlB}_{2}(\mathrm{C} 1)$ of load $\mathrm{v} / \mathrm{s}$ wear rate for $600 \mathrm{rpm}$ which as the minimum wear rate of $\left(1.9452 \times 10^{-6}\right.$ $\mathrm{g} / \mathrm{m}$ ) at load $20 \mathrm{~N}$, shows the remarkable trend with increasing load. That the higher temperature reveals the wear resistance due to the glaze formation that offers a protection and avoids further wear. Also due to combined addition of reinforced alloys to A413 alloy has resulted in minimum weight loss when compared to the individual addition (Al-5\% $\mathrm{Al}_{3} \mathrm{Ti}, \mathrm{Al}-3 \% \mathrm{AlB}_{2}, \mathrm{Al}-3 \% \mathrm{TiB}_{2}$ and $\mathrm{Al}-10 \% \mathrm{Al}_{4} \mathrm{Sr}$ reinforced intermetallic alloys) and in an treated condition.

\section{c. EFFECT OF SLIDING SPEED}

The effects of sliding speed on the wear rate of A413 alloy under constant normal pressure $\left(0.2497 \mathrm{~N} / \mathrm{mm}^{2}\right)$. From the figures it is clear that the wear rate of A413 alloy decreases with increase in sliding speeds $(0.942,1.884$ and $2.827 \mathrm{~m} / \mathrm{s}$ ) and temperature for all the cases studied. From Tables $3 \& 4$, figure $4, \mathrm{~A} 413+5 \% \mathrm{Al}_{3} \mathrm{Ti}$ (B1) of sliding Speed $\mathrm{v} / \mathrm{s}$ wear rate for $19.62 \mathrm{~N}$ has the maximum wear rate of $\left(9.1957 \times 10^{-6} \mathrm{~g} / \mathrm{m}\right)$ at sliding speed of $0.942 \mathrm{~m} / \mathrm{s}$ because of adding intermetallic particles and wear tends to more. $\mathrm{A} 413+3 \% \mathrm{TiB}_{2}(\mathrm{D} 1), \mathrm{A} 413+3 \% \mathrm{TiB}_{2}+10 \% \mathrm{Al}_{4} \mathrm{Sr}$ (F1) of sliding speed $\mathrm{v} / \mathrm{s}$ wear rate for $19.62 \mathrm{~N}$ as the sliding speed increases wear rate also increases. $\mathrm{A}_{413}+3 \% \mathrm{AlB}_{2}(\mathrm{C} 1)$ of sliding speed $\mathrm{v} / \mathrm{s}$ wear rate for $19.62 \mathrm{~N}$ as the minimum wear rate of $\left(1.9452 \times 10^{-6} \mathrm{~g} / \mathrm{m}\right)$ at sliding speed of $2.8274 \mathrm{~m} / \mathrm{s}$ shows the remarkable trend with increasing sliding speed. From Tables $3 \& 5$, figure 5 , A413 (A1) of sliding speed $\mathrm{v} / \mathrm{s}$ wear rate for $39.24 \mathrm{~N}$ as the maximum wear rate of $\left(12.3788 \times 10^{-6} \mathrm{~g} / \mathrm{m}\right)$ at sliding speed of $0.942 \mathrm{~m} / \mathrm{s}$ because they do not have intermetallic particles and wear tends to more. $\mathrm{A} 413+10 \% \mathrm{Al}_{4} \mathrm{Sr}(\mathrm{E} 1)$, $\mathrm{A} 413+3 \% \mathrm{TiB}_{2}+10 \% \mathrm{Al}_{4} \mathrm{Sr}(\mathrm{F} 1)$ of sliding speed $\mathrm{v} / \mathrm{s}$ wear rate for $39.24 \mathrm{~N}$, as the sliding speed increases the wear rate also increases. $\mathrm{A} 413+3 \% \mathrm{TiB}_{2}+10 \% \mathrm{Al}_{4} \mathrm{Sr}(\mathrm{F} 1)$ of sliding speed $\mathrm{v} / \mathrm{s}$ wear rate for $39.24 \mathrm{~N}$, as the minimum wear rate of $\left(1.6799 \times 10^{-6} \mathrm{~g} / \mathrm{m}\right)$ at sliding speed of $0.942 \mathrm{~m} / \mathrm{s}$ shows the remarkable trend with increasing sliding speed. From Tables $3 \& 6$, figure 6 , A413 (A1) of sliding speed $\mathrm{v} / \mathrm{s}$ wear rate for $58.86 \mathrm{~N}$ as the maximum wear rate of $\left(25.4650 \times 10^{-6} \mathrm{~g} / \mathrm{m}\right)$ at sliding speed of $0.942 \mathrm{~m} / \mathrm{s}$ because they do not have intermetallic particles and wear tends to more. $\mathrm{A} 413+3 \% \mathrm{TiB}_{2}+10 \% \mathrm{Al}_{4} \mathrm{Sr}(\mathrm{F} 1), \mathrm{A} 413+10 \% \mathrm{Al}_{4} \mathrm{Sr}(\mathrm{E} 1)$ of sliding speed $\mathrm{v} / \mathrm{s}$ wear rate for $58.86 \mathrm{~N}$, as the sliding speed increases the wear rate increases. $\mathrm{A} 413+5 \% \mathrm{Al}_{3} \mathrm{Ti}$ (B1) of sliding speed $\mathrm{v} / \mathrm{s}$ wear rate for $58.86 \mathrm{~N}$, as the minimum wear rate of $\left(2.5346 \times 10^{-6} \mathrm{~g} / \mathrm{m}\right)$ at sliding speed of $2.8274 \mathrm{~m} / \mathrm{s}$ shows the remarkable trend with increasing sliding speed. The reduction in wear with the increase in sliding speed may be possibly due to the increased contamination of sliding interface by oxide layer called glaze. The presence of 'glaze' reduces the chance of direct metallic contact and offer protection, therefore asperities interaction is reduced, which is a prerequisite for adhesive wear. Also at low sliding speeds, more time is available for the formation and growth of micro welds, which increases the force required to shear off the micro welds to maintain the relative motion, resulting in more wear rate. However, at higher speeds, there is less residential time for the growth of micro welds leading to lesser wear rate. So less weight loss was observed in A413 alloy containing combined reinforced intermetallic alloys when compared to as cast alloy.

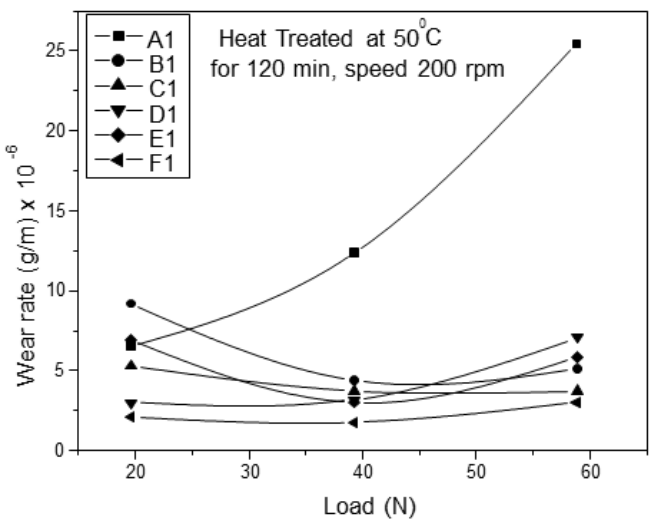

Figure 1. Load v/s Wear rate

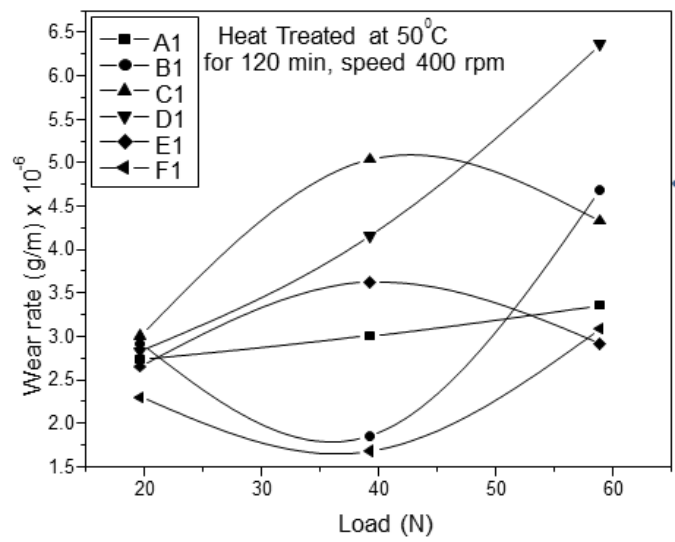

Figure 2. Load v/s Wear rate 
Table 4. Wear test data for specimens of various loads at heat treated at $500 \mathrm{C}$ for $120 \mathrm{~min}$ with constant speed (200 rpm), Track diameter $(90 \mathrm{~mm})$, Time $(5 \mathrm{~min})$, Sliding speed $(\mathrm{v})=0.942 \mathrm{~m} / \mathrm{s}$, Sliding distance $(\mathrm{L})=282.74 \mathrm{~m}$, (Pin diameter $=10 \mathrm{~mm})$

\begin{tabular}{|c|c|c|c|c|c|c|c|c|c|}
\hline $\begin{array}{l}\text { Alloy } \\
\text { Ref. }\end{array}$ & $\begin{array}{c}\text { Load } \\
\text { (N) }\end{array}$ & $\begin{array}{c}\text { Weight } \\
\text { Loss } \\
\text { (g) }\end{array}$ & $\begin{array}{c}\text { Volume } \\
\text { Loss } \\
\left(\mathrm{mm}^{3}\right)\end{array}$ & $\begin{array}{l}\text { Height } \\
\text { Loss } \\
(\mathrm{mm})\end{array}$ & $\begin{array}{c}\text { Linear wear } \\
\text { rate } \\
X 10^{-6} \\
(\mathrm{~mm} / \mathrm{m})\end{array}$ & $\begin{array}{l}\text { Wear } \\
\text { rate } \\
X 10^{-6} \\
(\mathrm{~g} / \mathrm{m})\end{array}$ & $\begin{array}{c}\text { Volume } \\
\text { Wear } \\
X^{-3} 10^{-3} \\
\left(\mathrm{~mm}^{3} / \mathrm{m}\right)\end{array}$ & $\begin{array}{c}\text { Specific Wear } \\
\text { Rate } \\
\text { X } 10^{-3} \\
\left(\mathrm{~mm}^{3} / \mathrm{N}-\mathrm{m}\right)\end{array}$ & $\begin{array}{c}\text { Wear } \\
\text { resistance } \\
X 10^{3} \\
\left(\mathrm{Nm} / \mathrm{mm}^{3}\right)\end{array}$ \\
\hline A1 & & 0.0018 & 0.7115 & 0.0090 & 31.8313 & 6.5431 & 2.5164 & 128.2568 & 7.7968 \\
\hline B1 & & 0.0026 & 1.0000 & 0.0127 & 44.9175 & 9.1957 & 3.5368 & 180.2650 & 5.5473 \\
\hline $\mathrm{C} 1$ & & 0.0015 & 0.5769 & 0.0073 & 25.8187 & 5.3052 & 2.0403 & 103.9908 & 9.6155 \\
\hline D1 & 19.62 & 0.0008 & 0.3269 & 0.0041 & 14.5009 & 3.0062 & 1.1561 & 58.9245 & 0.0169 \\
\hline E1 & & 0.0019 & 0.7500 & 0.0095 & 33.5997 & 6.8967 & 2.6526 & 135.1987 & 7.3965 \\
\hline F1 & & 0.0006 & 0.2307 & 0.0029 & 10.2567 & 2.1220 & 0.8159 & 41.5851 & 0.0240 \\
\hline A1 & & 0.0035 & 1.3461 & 0.0171 & 60.4795 & 12.3788 & 4.7609 & 121.3277 & 8.2421 \\
\hline B1 & & 0.0012 & 0.4807 & 0.0061 & 21.5745 & 4.4210 & 1.7001 & 43.3256 & 0.0230 \\
\hline $\mathrm{C} 1$ & & 0.0010 & 0.4038 & 0.0051 & 18.0377 & 3.7136 & 1.4281 & 36.3939 & 0.0274 \\
\hline D1 & 39.24 & 0.0009 & 0.3461 & 0.0044 & 15.5620 & 3.1831 & 1.2240 & 31.1926 & 0.0320 \\
\hline E1 & & 0.0008 & 0.3269 & 0.0041 & 14.5009 & 3.0062 & 1.1561 & 29.3476 & 0.0340 \\
\hline F1 & & 0.0005 & 0.1923 & 0.0024 & 8.4883 & 1.7684 & 0.6801 & 17.3318 & 0.0576 \\
\hline $\mathrm{A} 1$ & & 0.0072 & 2.7692 & 0.0352 & 124.4960 & 25.4650 & 9.7941 & 166.3965 & 6.0097 \\
\hline B1 & & 0.0014 & 0.5576 & 0.0070 & 24.7577 & 5.1283 & 1.9721 & 33.5049 & 0.0299 \\
\hline $\mathrm{C} 1$ & & 0.0010 & 0.4038 & 0.0051 & 18.0377 & 3.7136 & 1.4281 & 24.2626 & 0.0412 \\
\hline D1 & 58.86 & 0.0020 & 0.7692 & 0.0097 & 34.3071 & 7.0736 & 2.7205 & 46.2198 & 0.0216 \\
\hline E1 & & 0.0016 & 0.6346 & 0.0080 & 28.2945 & 5.8357 & 2.2444 & 38.1311 & 0.0262 \\
\hline F1 & & 0.0009 & 0.3269 & 0.0041 & 14.5009 & 3.0062 & 1.1561 & 19.6415 & 0.0509 \\
\hline
\end{tabular}

Table 5. Wear test data for specimens of various loads at heat treated at $50{ }^{0} \mathrm{C}$ for 120 min with constant speed (400 rpm), Track diameter $(90 \mathrm{~mm})$, Time $(5 \mathrm{~min})$, Sliding speed $(\mathrm{v})=0.942 \mathrm{~m} / \mathrm{s}$, Sliding distance $(\mathrm{L})=282.74 \mathrm{~m}$, (Pin diameter $=10 \mathrm{~mm})$.

\begin{tabular}{|c|c|c|c|c|c|c|c|c|c|}
\hline $\begin{array}{l}\text { Alloy } \\
\text { Ref. }\end{array}$ & $\begin{array}{l}\text { Load } \\
\text { (N) }\end{array}$ & $\begin{array}{c}\text { Weight } \\
\text { Loss } \\
\text { (g) }\end{array}$ & $\begin{array}{l}\text { Volume } \\
\text { Loss } \\
\left(\mathrm{mm}^{3}\right)\end{array}$ & $\begin{array}{l}\text { Height } \\
\text { Loss } \\
(\mathrm{mm})\end{array}$ & $\begin{array}{c}\text { Linear wear } \\
\text { rate } \\
X 10^{-6} \\
(\mathrm{~mm} / \mathrm{m})\end{array}$ & $\begin{array}{l}\text { Wear } \\
\text { rate } \\
X 10^{-6} \\
(\mathrm{~g} / \mathrm{m})\end{array}$ & $\begin{array}{l}\text { Volume } \\
\text { Wear } \\
X 10^{-3} \\
\left(\mathrm{~mm}^{3} / \mathrm{m}\right)\end{array}$ & $\begin{array}{c}\text { Specific Wear } \\
\text { Rate } \\
\text { X } 10^{-3} \\
\left(\mathrm{~mm}^{3} / \mathrm{N}-\mathrm{m}\right)\end{array}$ & $\begin{array}{c}\text { Wear } \\
\text { resistance } \\
X 10^{3} \\
\left(\mathrm{Nm} / \mathrm{mm}^{3}\right)\end{array}$ \\
\hline A1 & & 0.0021 & 0.8076 & 0.0102 & 12.0250 & 2.4757 & 0.9521 & 48.5270 & 0.0206 \\
\hline B1 & & 0.0019 & 0.0750 & 0.0009 & 1.0610 & 2.2989 & 0.0884 & 4.5056 & 0.2219 \\
\hline $\mathrm{C} 1$ & & 0.0016 & 0.0634 & 0.0008 & 0.9431 & 1.9452 & 0.0747 & 3.8073 & 0.2626 \\
\hline D1 & 19.62 & 0.0027 & 1.0576 & 0.0134 & 15.7970 & 3.2420 & 1.2468 & 63.5474 & 0.0157 \\
\hline E1 & & 0.0033 & 1.2692 & 0.0161 & 18.9807 & 3.8904 & 1.4962 & 76.2589 & 0.0131 \\
\hline F1 & & 0.0029 & 1.1346 & 0.0144 & 16.9765 & 3.4778 & 1.3376 & 68.1753 & 0.0146 \\
\hline A1 & & 0.0044 & 1.7115 & 0.0217 & 25.5826 & 5.2462 & 2.0177 & 51.4194 & 0.0194 \\
\hline B1 & & 0.0017 & 0.6538 & 0.0083 & 9.7850 & 2.0041 & 0.7707 & 19.6406 & 0.0509 \\
\hline $\mathrm{C} 1$ & & 0.0018 & 0.7115 & 0.0090 & 10.6103 & 2.1810 & 0.8388 & 21.3761 & 0.0467 \\
\hline D1 & 39.24 & 0.0035 & 1.3461 & 0.0171 & 20.1596 & 4.1262 & 1.5869 & 40.4408 & 0.0247 \\
\hline E1 & & 0.0037 & 1.4230 & 0.0181 & 21.3385 & 4.3620 & 1.6776 & 42.7522 & 0.0233 \\
\hline F1 & & 0.0030 & 1.1538 & 0.0146 & 17.2123 & 3.5367 & 1.3602 & 34.6636 & 0.0288 \\
\hline A1 & & 0.0033 & 1.2692 & 0.0176 & 20.7608 & 3.8904 & 1.4962 & 25.4196 & 0.0393 \\
\hline B1 & & 0.0021 & 0.8269 & 0.0105 & 12.3787 & 2.5346 & 0.9748 & 16.5613 & 0.0603 \\
\hline $\mathrm{C} 1$ & & 0.0022 & 0.8653 & 0.0110 & 12.9681 & 2.6525 & 1.0201 & 17.3309 & 0.0577 \\
\hline D1 & 58.86 & 0.0038 & 1.4615 & 0.0186 & 21.9280 & 4.4799 & 1.7229 & 29.2711 & 0.0341 \\
\hline E1 & & 0.0039 & 1.5192 & 0.0193 & 22.7532 & 4.6567 & 1.7910 & 30.4111 & 0.0328 \\
\hline F1 & & 0.0042 & 1.6153 & 0.0205 & 24.1679 & 4.9514 & 1.9043 & 32.3530 & 0.0309 \\
\hline
\end{tabular}


Table 6. Wear test data for specimens of various loads at heat treated at $50{ }^{\circ} \mathrm{C}$ for 120 min with constant speed $(600 \mathrm{rpm})$, Track diameter $(90 \mathrm{~mm})$, Time $(5 \mathrm{~min})$, Sliding speed $(\mathrm{v})=2.8274 \mathrm{~m} / \mathrm{s}$, Sliding distance $(\mathrm{L})=848.23 \mathrm{~m},($ Pin diameter $=10 \mathrm{~mm})$

\begin{tabular}{|c|c|c|c|c|c|c|c|c|c|}
\hline $\begin{array}{l}\text { Alloy } \\
\text { Ref. }\end{array}$ & $\begin{array}{l}\text { Load } \\
(\mathrm{N})\end{array}$ & $\begin{array}{c}\text { Weight } \\
\text { Loss } \\
\text { (g) }\end{array}$ & $\begin{array}{l}\text { Volume } \\
\text { Loss } \\
\left(\mathrm{mm}^{3}\right)\end{array}$ & $\begin{array}{l}\text { Height } \\
\text { Loss } \\
(\mathrm{mm})\end{array}$ & $\begin{array}{c}\text { Linear } \\
\text { wear rate } \\
\text { X10 } \\
(\mathrm{mm} / \mathrm{m})\end{array}$ & $\begin{array}{l}\text { Wear } \\
\text { rate } \\
X 10^{-6} \\
(\mathrm{~g} / \mathrm{m})\end{array}$ & $\begin{array}{c}\text { Volume } \\
\text { Wear } \\
\text { X } 10^{-3} \\
\left(\mathrm{~mm}^{3} / \mathrm{m}\right)\end{array}$ & $\begin{array}{c}\text { Specific } \\
\text { Wear Rate } \\
\text { X } 10^{-3} \\
\left(\mathrm{~mm}^{3} / \mathrm{N}-\mathrm{m}\right)\end{array}$ & $\begin{array}{c}\text { Wear } \\
\text { resistance } \\
X 10^{3} \\
\left(\mathrm{Nm} / \mathrm{mm}^{3}\right)\end{array}$ \\
\hline A1 & & 0.0015 & 0.5961 & 0.0075 & 13.4045 & 2.7410 & 1.0541 & 53.7257 & 0.0186 \\
\hline B1 & & 0.0016 & 0.3646 & 0.0046 & 8.2054 & 2.9178 & 0.6447 & 32.8593 & 0.0304 \\
\hline $\mathrm{C} 1$ & & 0.0017 & 0.6538 & 0.0083 & 14.7131 & 3.0062 & 1.1561 & 58.9245 & 0.0169 \\
\hline D1 & 19.62 & 0.0016 & 0.6153 & 0.0078 & 13.8466 & 2.8294 & 1.0881 & 55.4580 & 0.0180 \\
\hline E1 & & 0.0015 & 0.5769 & 0.0073 & 12.9801 & 2.6526 & 1.0201 & 51.9928 & 0.0192 \\
\hline F1 & & 0.0013 & 0.5000 & 0.0063 & 11.2470 & 2.2989 & 0.8842 & 45.0662 & 0.0221 \\
\hline A1 & & 0.0017 & 0.6538 & 0.0083 & 14.7131 & 3.0062 & 1.1561 & 29.4622 & 0.0339 \\
\hline B1 & & 0.0010 & 0.4038 & 0.0051 & 9.0719 & 1.8568 & 0.7140 & 18.1957 & 0.0549 \\
\hline $\mathrm{C} 1$ & & 0.0028 & 1.0961 & 0.0139 & 24.5808 & 5.0399 & 1.9383 & 49.3960 & 0.0202 \\
\hline D1 & 39.24 & 0.0023 & 0.9038 & 0.0115 & 20.3367 & 4.1557 & 1.5982 & 40.7288 & 0.0245 \\
\hline E1 & & 0.0020 & 0.7884 & 0.0100 & 17.8640 & 3.6252 & 1.3942 & 35.5300 & 0.0281 \\
\hline F1 & & 0.0009 & 0.3653 & 0.0046 & 8.2054 & 1.6799 & 0.6459 & 16.4602 & 0.0607 \\
\hline A1 & & 0.0019 & 0.7307 & 0.0092 & 16.4285 & 3.3599 & 1.2921 & 21.9520 & 0.0455 \\
\hline B1 & & 0.0026 & 1.0192 & 0.0192 & 34.1126 & 4.6862 & 1.8023 & 30.6201 & 0.0326 \\
\hline $\mathrm{C} 1$ & & 0.0024 & 0.9423 & 0.0119 & 21.0440 & 4.3326 & 1.6663 & 28.3095 & 0.0353 \\
\hline D1 & 58.86 & 0.0036 & 1.3846 & 0.0176 & 31.1240 & 6.3662 & 2.4485 & 41.5987 & 0.0240 \\
\hline E1 & & 0.0016 & 0.6346 & 0.0080 & 14.2710 & 2.9178 & 1.1222 & 19.0655 & 0.0524 \\
\hline F1 & & 0.0017 & 0.6730 & 0.0056 & 9.9915 & 3.0947 & 1.1901 & 20.2191 & 0.0494 \\
\hline
\end{tabular}

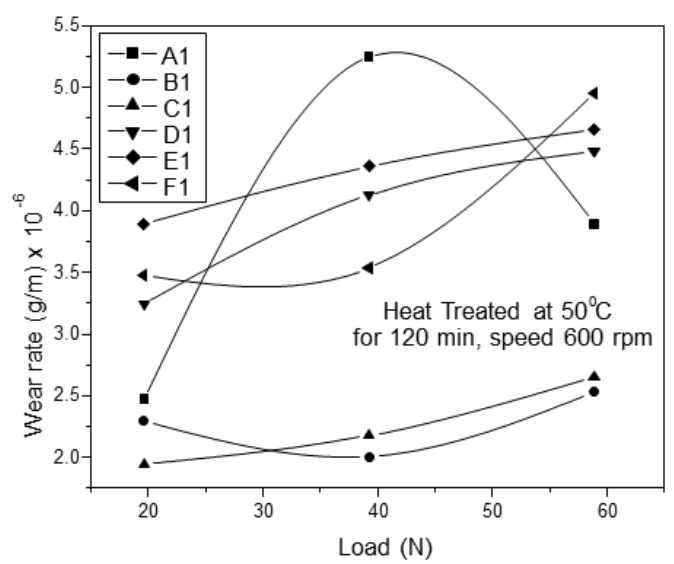

Figure 3. Load v/s Wear rate

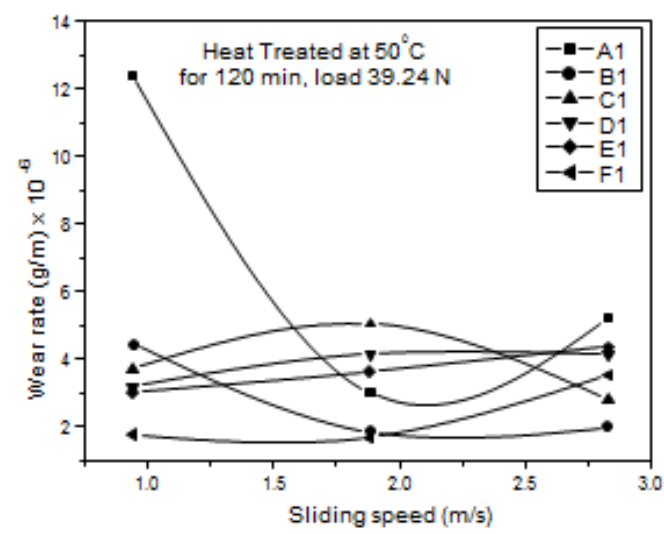

Figure 5. Sliding speed v/s Wear rate

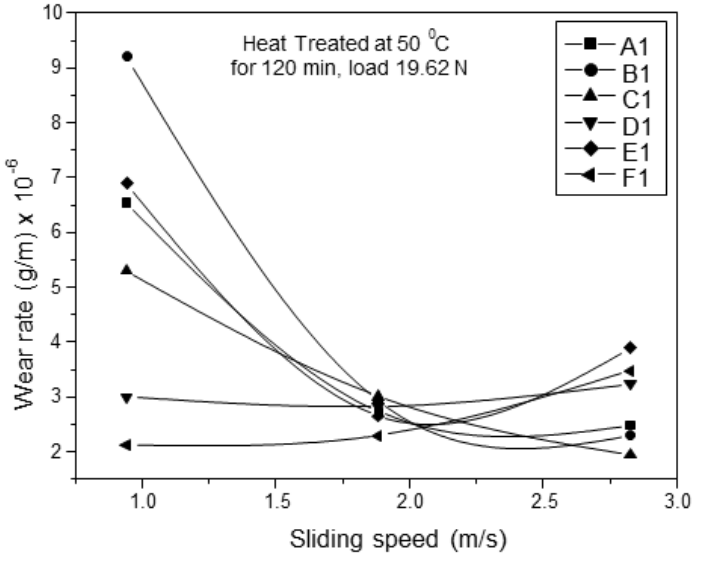

Figure 4. Sliding speed v/s Wear rate

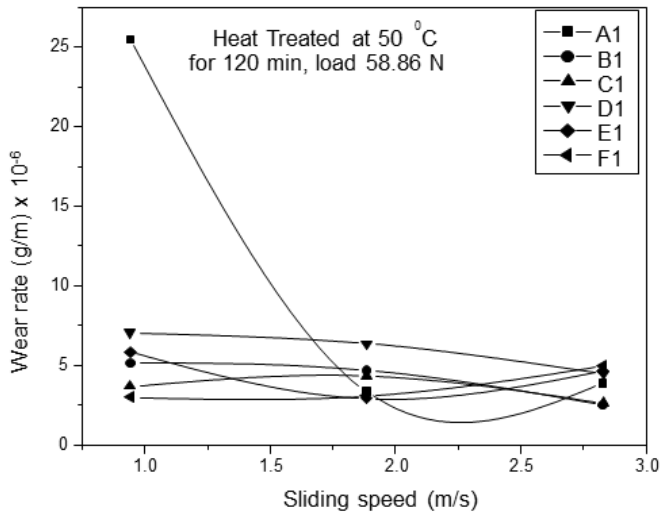

Figure 6. Sliding speed v/s Wear rate 
From Table $3 \&$ figure 1 it is observe that A413 (A1) of load v/s wear rate for $200 \mathrm{rpm}$ has the maximum wear rate $\left(25.4650 \times 10^{-6} \mathrm{~g} / \mathrm{m}\right)$ at load $60 \mathrm{~N}$. A413 $+5 \% \mathrm{Al}_{3} \mathrm{Ti}(\mathrm{B} 1), \mathrm{A} 413+3 \% \mathrm{AlB}_{2}(\mathrm{C} 1), \mathrm{A} 413+10 \% \mathrm{Al}_{4} \mathrm{Sr}(\mathrm{E} 1)$ of load v/s wear rate for $200 \mathrm{rpm}$, shows that wear rate decreases slightly as load increases. $\mathrm{A} 413+3 \% \mathrm{TiB}_{2}+10 \% \mathrm{Al}_{4} \mathrm{Sr}(\mathrm{F} 1)$ of load $\mathrm{v} / \mathrm{s}$ wear rate for $200 \mathrm{rpm}$ has minimum wear rate $\left(1.7684 \times 10^{-6} \mathrm{~g} / \mathrm{m}\right)$ at load $60 \mathrm{~N}$, shows the remarkable trend with increasing load. From Table $3 \&$ figure 2, we seen that, $\mathrm{A} 413+3 \% \mathrm{TiB}_{2}(\mathrm{D} 1)$ of load v/s wear rate for $400 \mathrm{rpm}$ has maximum wear rate $\left(6.3662 \times 10^{-6} \mathrm{~g} / \mathrm{m}\right)$ at load $60 \mathrm{~N}$. A413 (A1), A413+5\% $\mathrm{Al}_{3} \mathrm{Ti}(\mathrm{B} 1), \mathrm{A} 413+3 \% \mathrm{TiB}_{2}+10 \% \mathrm{Al}_{4} \mathrm{Sr}$ (F1) of load v/s wear rate for $400 \mathrm{rpm}$ has the minimum wear rate at $20 \mathrm{~N}$ as the load increases to $40 \mathrm{~N}$ wear rate slightly increases. $\mathrm{A} 413+3 \% \mathrm{TiB}_{2}+10 \% \mathrm{Al}_{4} \mathrm{Sr}(\mathrm{F} 1)$ of load $\mathrm{v} / \mathrm{s}$ wear rate for $400 \mathrm{rpm}$ has minimum wear rate $\left(1.6799 \times 10^{-6} \mathrm{~g} / \mathrm{m}\right)$ at load $20 \mathrm{~N}$. shows that the remarkable trend with increasing load

From Table 3, figure 3, we seen that, A413 (A1) load v/s wear rate for $600 \mathrm{rpm}$ has maximum wear rate $\left(5.2462 \times 10^{-}\right.$ $\left.{ }^{6} \mathrm{~g} / \mathrm{m}\right)$ at load $40 \mathrm{~N}$. A413+3\%AlB $2(\mathrm{C} 1), \mathrm{A}_{413}+3 \% \mathrm{TiB}_{2}(\mathrm{D} 1), \mathrm{A} 413+10 \% \mathrm{Al}_{4} \mathrm{Sr}(\mathrm{E} 1), \mathrm{A} 413+3 \% \mathrm{TiB}_{2}+10 \% \mathrm{Al}_{4} \mathrm{Sr}$

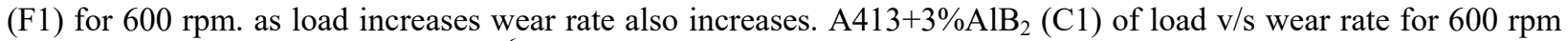
has minimum wear rate $\left(1.9452 \times 10^{-6} \mathrm{~g} / \mathrm{m}\right)$ at load $20 \mathrm{~N}$ shows the remarkable trend with increasing load. From Table 4, 5 \& 6, figure 4, we got $\mathrm{A} 413+5 \% \mathrm{Al}_{3} \mathrm{Ti}$ (B1) of sliding speed $\mathrm{v} / \mathrm{s}$ wear rate for $19.62 \mathrm{~N}$ has the maximum wear rate $\left(9.1957 \times 10^{-6} \mathrm{~g} / \mathrm{m}\right)$ at sliding speed of $0.942 \mathrm{~m} / \mathrm{s}$. A413+3\% $\mathrm{TiB}_{2}(\mathrm{D} 1), \mathrm{A} 413+3 \% \mathrm{TiB}_{2}+10 \% \mathrm{Al}_{4} \mathrm{Sr}(\mathrm{F} 1)$ of sliding speed $\mathrm{v} / \mathrm{s}$ wear rate for $19.62 \mathrm{~N}$, as the sliding speed increases wear rate also increases. $\mathrm{A}_{413}+3 \% \mathrm{AlB}_{2}(\mathrm{C} 1)$ of sliding speed $\mathrm{v} / \mathrm{s}$ wear rate for $19.62 \mathrm{~N}$ has the minimum wear rate $\left(1.9452 \times 10^{-6} \mathrm{~g} / \mathrm{m}\right)$ at sliding speed of 2.8274 $\mathrm{m} / \mathrm{s}$, shows that remarkable trend with increasing sliding speed.

From Table 4, $5 \& 6$, figure 5, it seen that A413 (A1) of sliding speed $\mathrm{v} / \mathrm{s}$ wear rate for $39.24 \mathrm{~N}$ has the maximum wear rate $\left(12.3788 \times 10^{-6} \mathrm{~g} / \mathrm{m}\right)$ at sliding speed of $0.942 \mathrm{~m} / \mathrm{s}$. A413+10\% $\mathrm{Al}_{4} \mathrm{Sr}(\mathrm{E} 1), \mathrm{A} 413+3 \% \mathrm{TiB}_{2}+10 \% \mathrm{Al}_{4} \mathrm{Sr}(\mathrm{F} 1)$ of sliding speed $\mathrm{v} / \mathrm{s}$ wear rate for $39.24 \mathrm{~N}$, as the sliding speed increases the wear rate also increases. $\mathrm{A} 413+3 \% \mathrm{TiB}_{2}+10 \% \mathrm{Al}_{4} \mathrm{Sr} \quad(\mathrm{F} 1)$ of the sliding speed $\mathrm{v} / \mathrm{s}$ wear rate for $39.24 \mathrm{~N}$ has the minimum wear rate $\left(1.6799 \times 10^{-6} \mathrm{~g} / \mathrm{m}\right)$ at sliding speed $0.942 \mathrm{~m} / \mathrm{s}$, shows that remarkable trend with increasing sliding speed. From Table 4, 5 \& 6, figure 6, we observe that A413 (A1) of sliding speed $\mathrm{v} / \mathrm{s}$ wear rate for $58.86 \mathrm{~N}$ has the maximum wear rate $\left(25.4650 \times 10^{-6} \mathrm{~g} / \mathrm{m}\right)$ at sliding speed of $0.942 \mathrm{~m} / \mathrm{s}$. A $413+3 \% \mathrm{TiB}_{2}+10 \% \mathrm{Al}_{4} \mathrm{Sr}(\mathrm{F} 1), \mathrm{A}_{413}+10 \% \mathrm{Al}_{4} \mathrm{Sr}(\mathrm{E} 1)$ of sliding speed $\mathrm{v} / \mathrm{s}$ wear rate for $58.86 \mathrm{~N}$, as the sliding speed increase the wear rate increases. $\mathrm{A} 413+5 \% \mathrm{Al}_{3} \mathrm{Ti}(\mathrm{B} 1)$ of sliding speed $\mathrm{v} / \mathrm{s}$ wear rate for $58.86 \mathrm{~N}$ has the minimum wear rate $\left(2.5346 \times 10^{-6} \mathrm{~g} / \mathrm{m}\right)$ at sliding speed $2.8274 \mathrm{~m} / \mathrm{s}$ shows that remarkable trend with increasing sliding speed.

\section{CONCLUSION}

The intermetallic particles influences on wear rate. The higher temperature reveals the wear resistance due to the glaze formation that offers a protection and avoids further wear. Due to combined reinforced alloys to A413 alloy has resulted in minimum weight loss when compared to the individual addition $\left(\mathrm{Al}-5 \% \mathrm{Al}_{3} \mathrm{Ti}, \quad \mathrm{Al}-3 \% \mathrm{AlB}{ }_{2}, \mathrm{Al}-\right.$ $3 \% \mathrm{TiB}_{2}$ and $\mathrm{Al}-10 \% \mathrm{Al}_{4} \mathrm{Sr}$ reinforced intermetallic alloys) and in heat treated conditions.

\section{REFERENCES}

[1] Saheb. N., Laoui. T., Daud. A. R., Harun. M., Radiman. S. and Yahaya. R., Wear, Vol.249, 2001, p 656-662.

[2] Gruzleski J. E. and B. M Closet, "Liquid treatment to Al-Si alloys", AFS, Illions, 1990, p 1-254.

[3] McCartney. D. G., Int. Mater. Rev., 34, 1989, p 247-260.

[4] Kori. S. A., Murty. B. S., and Chakraborty. M., Mater. Sc. Engg. A, Vol. 280, 2000, p 94-104.

[5] Murty. B. S, Kori. S. A. and M. Chakraborty., Inter. Mater. Rev., Vol. 47, 2002, p 3-29.

[6] Katsuta. M., Oodoshi. K. and Kohara. S., Proce. $6^{\text {th }}$ Inter. Conf. on Aluminium alloys (ICAA-6), 1998, Vol.3, p 1945-1950.

[7] Shivanath. R., Sengupta. P. K., Eyre. T. S., British Foundrymen, Vol.79, 1977, p 349-356.

[8] Somi Reddy. A., Pramila Bai. B. N., Murthy. K. S. S. and Biswas. S. K., Wear, Vol.181-183, 1995, p 658-667.

[9] Somi Reddy. A, Pramila Bai. B. N., Murthy. K. S. S. and Biswas. S. K., Wear, Vol. 171, 1994, p 115-127

[10] Torbian. H., Pathak. J. P. and Tiwari. S. N., Wear, Vol.172, 1994, p 49-58.

[11] Pramila Bai. B. N. and Biswas. S. KWear, Vol.120, 1987, p 61-74.

[12] Pathak. J. P. and Mohan. S, Indian Foundry Journal, 1998, p 1-9.

[13] Sarkar. A. D., Wear, Vol.31, 1975, p 331-343.

[14] Clarke. J. and Sarkar. A. D., Wear, Vol. 54, 1979, p 7-16.

[15] Eyre. T. S., Met. Technol, Vol.11, 1984, p 81-90.

[16] Mohd Harun, Talib. I. A. and Daud. A.R., Wear, Vol.194, 1996, p 54-59.

[17] Dasgupta. A., Bose. S. K., J. Mater. Sci. Lett., Vol.14, 1995, p1661-1663.

[18] Martin A, Martinez MA, Llorca J., Wear, Vol. 193, 1996, p 169-179.

[19] Pauschitz A, Roy M, Franek F., Tribologia, Vol.188, 2003, p 127.

[20] Roy M, Pauschitz A, Wernisch J, Franek F., Materials and Corrosion, Vol.55, 2004, p 259-273.

[21] Pauschitz A, Roy M, Franek F., Tribology International, Vol.41, 2008, p 584-602. 
[22] Stott FH., Tribology International, Vol. 35, 2002, p 489-495.

[23] Welsh NC., Journal of Applied Physics, Vol.28, 1957, p 960-968.

[24] Yongzhong Z, Guoding Z., Materials and Design, Vol.27, 2006, p 79-84.

[25] Venkateswarlu K, Pathak LC, Ray AK, Goutam D, Verma PK, Kumar M, Ghosh RN., Material Science Engineering, Vol.383A, 2004, p 374-80.

[26] Prasad BK, Venkateswarlu K, Modi OP, Jha AK, Das S, Dasgupta R., Metallurgical and Materials Transactions, Vol.29A, 1998, p 27472752.

[27] Prasad BK, Venkateswarlu K, Modi OP, Yegneswaran AH., Journal of Materials Science Letters, Vol.15, 1996, p 1773-1776.

[28] Rajinikanth V, Venkateswarlu K., Tribology International, Vol.44, 2011, p1711-1719.

[29] H.R. Manohara, T.M.Chandrashekharaiah, K.Venkateswarlu, S.A.Kori., Tribology International, Vol.51, 2012, p 54-60.

[30] O. El Sebaie ${ }^{\mathrm{a}}$, A.M. Samuel ${ }^{\mathrm{a}}$, F. H. Samuel ${ }^{\mathrm{a}, *}$, H.W. Doty ${ }^{\mathrm{b}}$, "The effects of mischmetal, cooling rate and heat treatment on the hardness of A319.1, A356.2 and A412.1 Al-Si casting alloys" Material Science and Engineering A 486 (2008) 241-252.

[31] M. A. Moustafa, F. H. Samuel, H. W. Doty , "Effect of solution heat treatment and additives on the microstructure of Al-Si (A413.1) automotive alloys" Material science 38(2003), pp 4507-4522 